\title{
Survival of Verapamil-Poisoned Rats Treated with Triiodothyronine
}

\author{
Michael J. Lynch • Kenneth D. Katz • \\ Clifton W. Callaway $•$ Eric S. Logue
}

Published online: 17 March 2010

(C) American College of Medical Toxicology 2010

\begin{abstract}
Life-threatening toxicity due to calcium channel blocker ingestion is commonly encountered by emergency medicine physicians and toxicologists. Despite a vast array of research on its treatment, results have proven inconsistent. The goal of this study is to evaluate potential vasopressor effects of triiodothyronine (T3) in rats poisoned with verapamil. Following anesthesia and intubation, ten Sprague-Dawley rats were given intravenous verapamil infusion of $10 \mathrm{mg} / \mathrm{kg} / \mathrm{h}$. This dose was titrated until a mean arterial pressure (MAP) of 50-55 $\mathrm{mmHg}$ was achieved and maintained for a period of at least $5 \mathrm{~min}$. The verapamil infusion was then maintained at that rate. Five rats were randomized to receive a $\mathrm{T} 3$ bolus of $0.4 \mathrm{mcg} / \mathrm{kg}$ preceding an infusion of $1.5 \mathrm{mcg} / \mathrm{kg} /$ day which was doubled every 2 min until any of the following endpoints: systolic blood pressure of $100 \mathrm{mmHg}$, an elapsed time of $60 \mathrm{~min}$, or death. The other five received an equal volume of normal saline solution. The primary outcome measure was survival with secondary outcomes of MAP and heart rate. The T3 group did have a slightly longer, yet not statistically significant, average time to cessation of electrical activity$30.0 \pm 14.4 \mathrm{~min}$ versus $23.8 \pm 9.5 \mathrm{~min}$ in the placebo group. Average MAP decreased nearly identically in the two groups. Heart rates were not reliable indicators of toxicity in this rat model as there was little decrease until
\end{abstract}

M. J. Lynch $(\bowtie) \cdot$ K. D. Katz $\cdot$ C. W. Callaway $\cdot$ E. S. Logue Department of Emergency Medicine,

University of Pittsburgh Medical Center,

Pittsburgh, PA, USA

e-mail: lynchmj@upmc.edu

M. J. Lynch · K. D. Katz

Division of Medical Toxicology,

University of Pittsburgh Medical Center,

Pittsburgh, PA, USA immediately prior to death in most animals. Despite significant variability in toxicity among individual animals, no statistically significant difference in survival time, heart rate, or MAP was found between groups treated with $\mathrm{T} 3$ and those receiving saline.

Keywords Calcium channel blocker - Toxicity · Verapamil · Triiodothyronine . Thyroid hormone

\section{Introduction}

Calcium channel blockers (CCBs) account for approximately $40 \%$ of cardiovascular drug exposures reported to the American Association of Poison Centers and account for greater than $65 \%$ of deaths from cardiovascular medications [1]. Despite implementation of multiple therapeutic and resuscitative modalities, the mortality from $\mathrm{CCBs}$ remains unacceptably high. Among the three classes of CCBs, the diphenylakylamines are the most toxic and have been associated with highest morbidity and mortality.

The CCBs act by depressing the activation, inactivation, and recovery of L-type voltage-operated calcium channels in the heart and smooth muscle of coronary and peripheral vasculature causing reduced intracellular calcium. This action results in depressed chronotropy and inotropy as well as peripheral vasodilation. Current therapeutic strategies for $\mathrm{CCB}$ toxicity have produced inconsistent clinical effects [2-7]. Thyroid hormone, however, may represent a novel therapeutic approach.

The active form of thyroid hormone, triiodothyronine (T3), exerts its contractile effects on cardiac myocytes via nuclear thyroid hormone receptors causing increased expression of calcium adenosine triphosphatase of the sarcoplasmic reticulum and alpha-myosin heavy chain. 
The chronotropic activity of thyroid hormone is related to its activity on specific ion channel proteins in the sinoatrial node, including the sodium-calcium exchanger protein, the L-type and T-type calcium channel, and ryanodine channel $[8,9]$. Thyroid hormone has also been shown to increase gene expression of $\mathrm{Na}^{+}, \mathrm{K}^{+}$-ATPase [10].

Both in vitro studies and several case reports have illustrated thyroid hormone's stimulatory effect on cardiac myocytes and its vasopressor activity [11, 12]. Retrospective reviews have found decreased vasopressor requirements in organ donor populations with myocardial depression and hypotension treated with T3 [13-16]. Moreover, prospective studies of T3 treatment have shown improved cardiovascular outcomes following coronary artery bypass grafting (CABG) $[17,18]$. These studies provide the necessary rationale to examine the potential efficacy of $\mathrm{T} 3$ as an antidote for CCB toxicity.

\section{Materials and Methods}

Study protocol and drugs were approved by the University of Pittsburgh Institutional Animal Care and Use Committee (IACUC). Maximum of ten rats were chosen, in accordance with IACUC policies directing the allowable number of animal subjects used in a pilot study. A preliminary power calculation for time to cessation of pulse was not performed. Based on previously published data by Tuncok et al., a power calculation suggested that $80 \%$ power could be achieved with five animals in each group if an average difference in mean arterial pressure (MAP) was $10 \mathrm{mmHg}$ at the completion of the protocol, assuming standard deviations of $5 \mathrm{mmHg}$ (5.1\% of average baseline MAP of $103 \mathrm{mmHg}$ ) [19]. With standard deviations of $10 \mathrm{mmHg}$, a difference in MAP of $20 \mathrm{mmHg}$ would need to be seen [20, 21]. However, in that study, both control and treatment groups had improvement in MAP. Our protocol anticipated continued decline in blood pressure in the control group.

Ten Sprague-Dawley rats weighing 300-360 g received a subcutaneous injection of ketamine $(40 \mathrm{mg} / \mathrm{kg})$ and xylazine $(10 \mathrm{mg} / \mathrm{kg})$. The analgesic buprenorphine $(0.2 \mathrm{mg} / \mathrm{kg})$ was administered subcutaneously after loss of the righting reflex. Rats were not paralyzed and were monitored for signs of lightening sedation. An additional dose of buprenorphine would have been given for signs of awakening. However, no animal in either group required additional anesthesia. Inhaled anesthetics were not administered. The anesthetized rats were endotracheally intubated with a 14-gauge plastic catheter (Angiocath) and then mechanically ventilated $(9 \mathrm{cc} / \mathrm{kg}, 43$ respirations $/ \mathrm{min}$, SAR830 Series Small Animal Ventilator, CWE, Inc., Ardmore, PA, USA). When deeply anesthetized, an incision was made over both inguinal creases, and the femoral blood vessels were exposed by blunt dissection. One femoral arterial and bilateral venous catheters (one for verapamil and $\mathrm{T} 3$ infusions, respectively) made from PE-50 tubing were placed and secured with 3-0 silk ties. The femoral arterial catheter was used for arterial blood gas sampling as well as continuous blood pressure monitoring. Baseline arterial blood gas requiring $0.2 \mathrm{cc}$ 's was drawn. Continuous cardiac monitoring was provided via electrocardiogram (ECG) leads.

Both groups were given intravenous verapamil starting with an infusion rate of $10 \mathrm{mg} / \mathrm{kg} / \mathrm{h}$. A previous study by Tuncok et al. indicated that a 10-min infusion of verapamil at a dose of $15 \mathrm{mg} / \mathrm{kg} / \mathrm{h}$ decreased rat MAP by roughly $50 \%$ [19]. As the animals' blood pressure approached a MAP of $55 \mathrm{mmHg}$, the dose was halved and then titrated until a MAP of $50-55 \mathrm{mmHg}$ was maintained for a period of $5 \mathrm{~min}$ at the same infusion rate. The approach of titrating verapamil dosing to a temporary steady state was adapted from the protocol used in canine poisoning by Bania et al. [6]. The verapamil infusion was maintained at that rate throughout the experiment to mimic continual absorption in a massive overdose. Discontinuation of verapamil administration by Tuncok et al. resulted in spontaneous improvement in both the control and treatment groups [19]. Our protocol was designed to replicate the course of lethal human verapamil toxicity as closely as possible. Continued verapamil administration allowed time to pulselessness to be used as the primary endpoint. Additionally, potential benefits of treatment would be more significant if a divergence in hemodynamic measures was found between groups rather than small differences in improvement following cessation of verapamil. Baseline rat blood pressures are similar to humans'. A MAP of $55 \mathrm{mmHg}$ was chosen to simulate significant toxicity in a human patient. In human $\mathrm{CCB}$ toxicity, a MAP of $55 \mathrm{mmHg}$ or below would certainly necessitate aggressive treatment. Continuous heart rate, ECG tracing, systolic and diastolic pressures, and MAP were recorded (Chart, v.5.3, ADInstruments, Castle Hill, Australia). Following achievement of

Table 1 Baseline data for both the experimental and control group

\begin{tabular}{lcc}
\hline & T3 group & Saline group \\
\hline Weight $(\mathrm{g})$ & $333.4 \pm 21.0$ & $328.2 \pm 20.9$ \\
Temperature $\left({ }^{\circ} \mathrm{C}\right)$ & $37.0 \pm 0.4$ & $37.1 \pm 0.4$ \\
Heart rate $(\mathrm{bpm})$ & $250.4 \pm 16.0$ & $263.6 \pm 22.0$ \\
Mean arterial pressure $(\mathrm{mmHg})$ & $111.6 \pm 19.7$ & $122.0 \pm 10.5$ \\
$\mathrm{pH}$ & $7.40 \pm 0.04$ & $7.39 \pm 0.03$ \\
Ionized $\mathrm{Ca}^{2}+$ & $1.37 \pm 0.04$ & $1.35 \pm 0.01$ \\
Glucose & $312.2 \pm 41.6$ & $368.8 \pm 66.2$ \\
Lactate & $1.95 \pm 0.37$ & $1.94 \pm 0.21$ \\
\hline
\end{tabular}

No significant baseline differences were noted between groups 
Fig. 1 Kaplan-Meier survival curve. The difference in survival between groups was not statistically significant. Group 1 represents the treatment group and group 2 is the control group
Kaplan-Meier survival estimates, by group

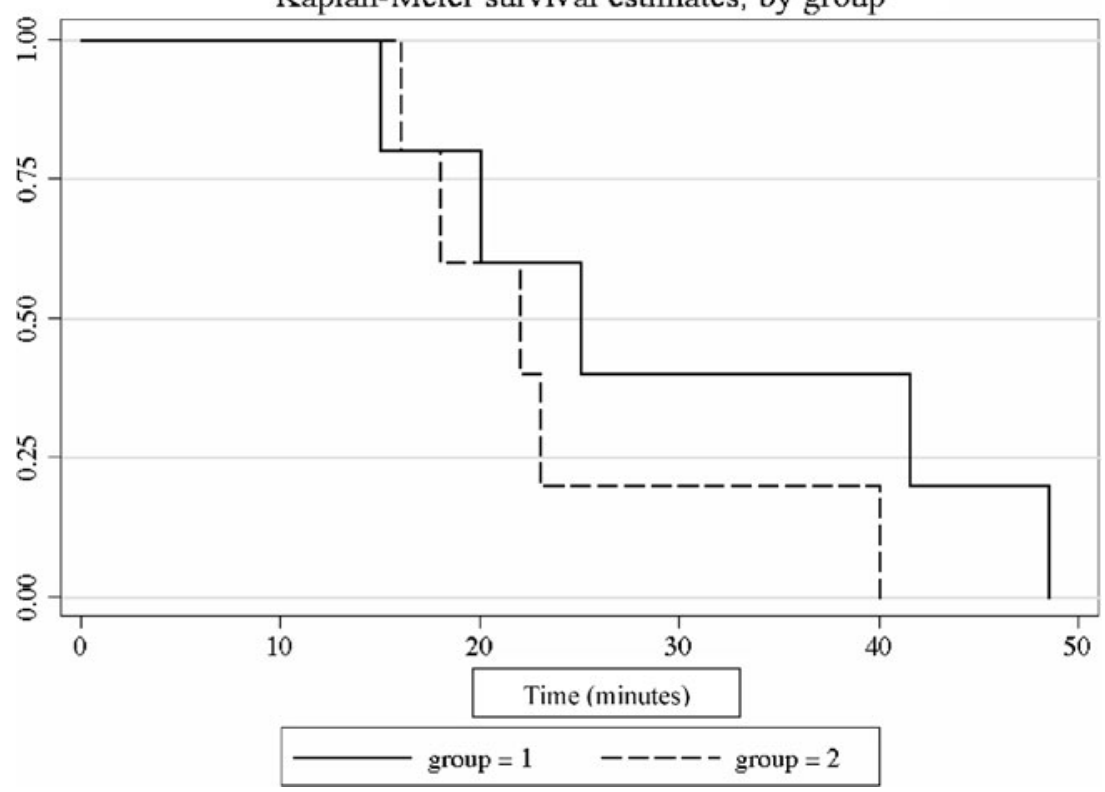

target MAP, five rats were randomized to receive a T3 bolus of $0.4 \mathrm{mcg} / \mathrm{kg}$ preceding an infusion of $1.5 \mathrm{mcg} / \mathrm{kg} /$ day which was doubled every 2 min until any of the following endpoints: systolic blood pressure of $100 \mathrm{mmHg}$, an elapsed time of $60 \mathrm{~min}$, or death. The other five received an equal volume of normal saline solution. The T3 dose was based on the Children's Hospital of Pittsburgh Drug Formulary for Triostat (T3) treatment of hypotension in the setting of pediatric heart failure and organ preservation. The infusion rate was doubled every $2 \mathrm{~min}$ to evaluate potential dose response at unusually high doses and to simulate titration of therapy in the clinical setting. Infusions were delivered via infusion pump (BS-8000 Programmable Infusion Pump, Braintree Scientific, Braintree, MA, USA).
The primary outcome measure was time to cessation of pulse. Kaplan-Meier curve compared to a log-rank test was used to evaluate survival times. Changes in heart rate and MAP were continuously recorded. Heart rate and MAP were secondary outcome measures evaluated by a general linear model (GLM) due to the number of data points recorded. Subsequent blood testing could not be obtained due to hemodynamic instability associated with phlebotomy.

\section{Results}

Baseline weight, arterial blood gas, glucose, and ionized calcium levels were obtained for each animal and are
Fig. 2 Mean arterial pressure of each group following initiation of T3 or placebo administration. No significant difference between groups was noted

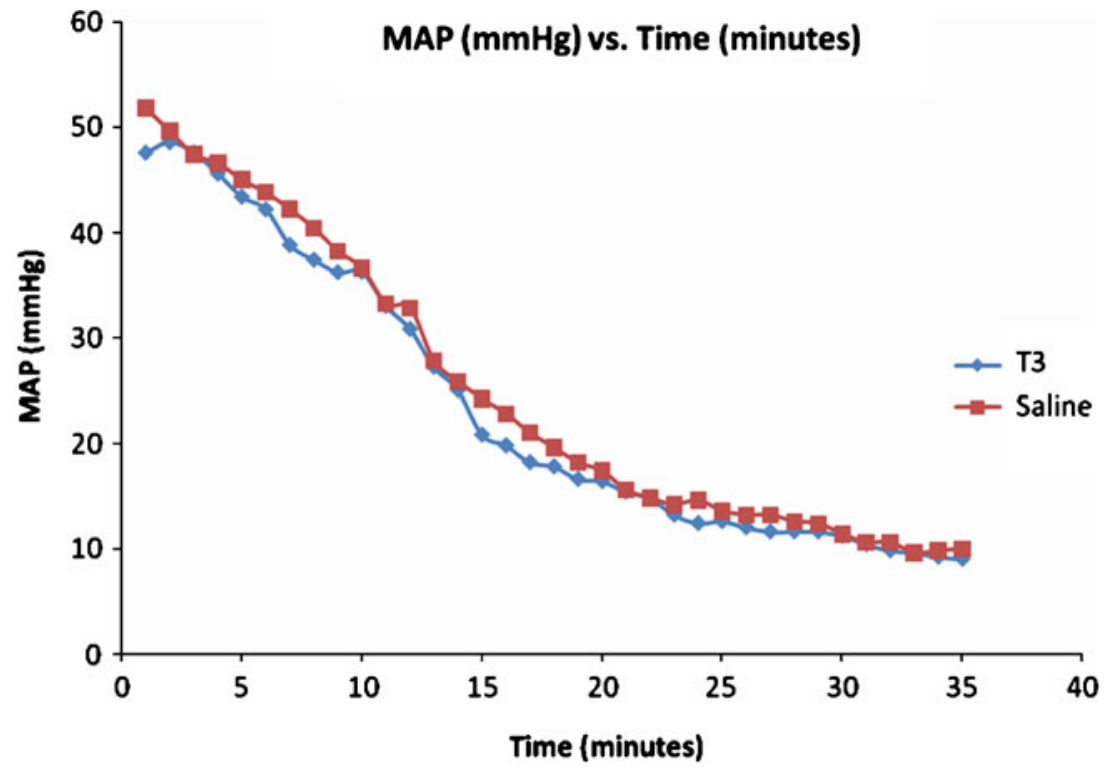


Fig. 3 Heart rates of rats in the T3 treatment group versus time. The response of heart rate to verapamil infusion was inconsistent between animals. Animals developed asystole precipitously

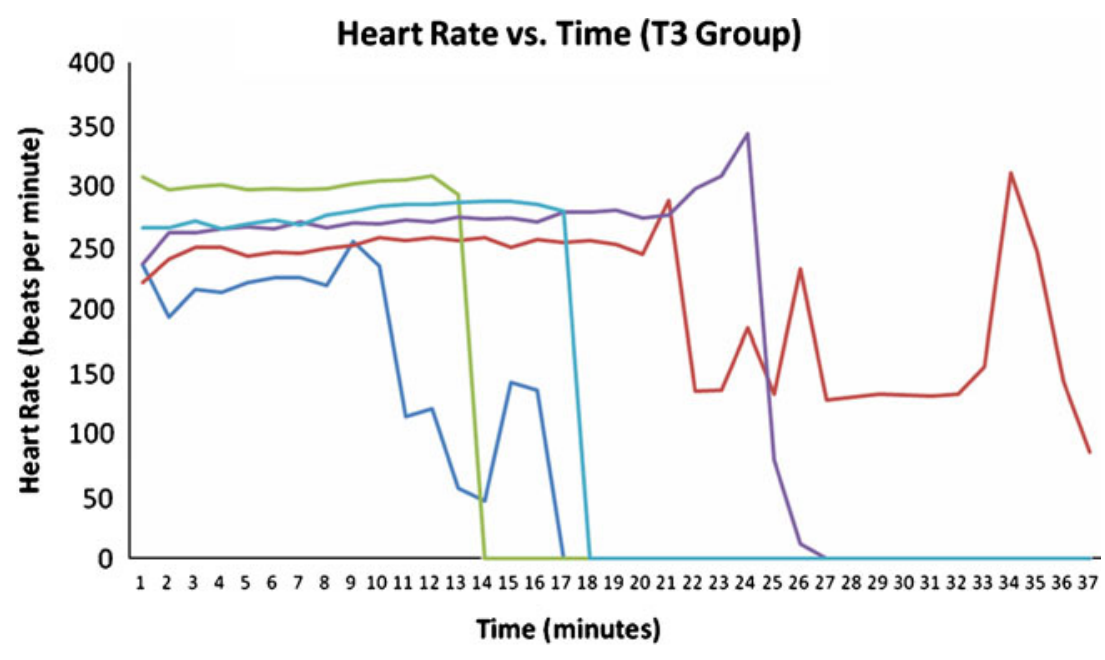

summarized in Table 1; there were no significant differences between the study and control groups. Despite significant variability in toxicity among individual animals, no statistically significant difference in survival time, heart rate, or MAP was found between groups. The T3 group did have a slightly longer, yet not statistically significant, average time to cessation of electrical activity-30.0土 $14.4 \mathrm{~min}$ versus $23.8 \pm 9.5 \mathrm{~min}$ in the placebo group. Kaplan-Meier curve (Fig. 1) compared to a log-rank test resulted in a value of $1.24(p=0.265)$.

Neither MAP nor heart rate differed significantly between groups. As illustrated in Fig. 2, mean MAP of each group decreased nearly identically. GLM evaluation of the data revealed an effect of time on MAP, but no effect of group $\left(F_{(1,8)}=0.11, p=0.75\right)$. GLM evaluation of heart rate also revealed no effect of group $\left(F_{(1,8)}=0.04, p=0.839\right)$. Interestingly, rats in both groups maintained heart rates with little to no change until shortly before death at which point heart rates decreased precipitiously (Figs. 3 and 4) Analysis of heart rate prior to the death of the first rat does not reveal an effect of time. Based on the data collected, heart rate does not serve as a reliable marker of toxicity in rat models of verapamil compared to MAP which steadily decreases as toxicity worsens. The total amount of verapamil infused was nearly identical between the two groups $-157 \pm 52 \mu \mathrm{l}$ in the T3 group versus $149 \pm 46 \mu 1$ in the control group.

\section{Discussion}

Thyroid hormone has been used effectively for cardiovascular support in both animal and human models; however, it had not been applied to acute, profound drug-induced cardiovascular toxicity. Timek et al. showed that T3 reverses depressed contractile performance following high-dose epinephrine exposure in isolated porcine myocardium despite reduced intracellular calcium concentrations leading to the conclusion that T3 may sensitize the cardiac myocyte contractile apparatus to calcium [11]. Another in vitro study performed on porcine cardiac myocytes by Walker et al. showed that T3 caused a dosedependent increase in myocyte contractile performance
Fig. 4 Rates of rats in the control treatment group versus time. As in the T3 group, heart rates dropped precipitously prior to death

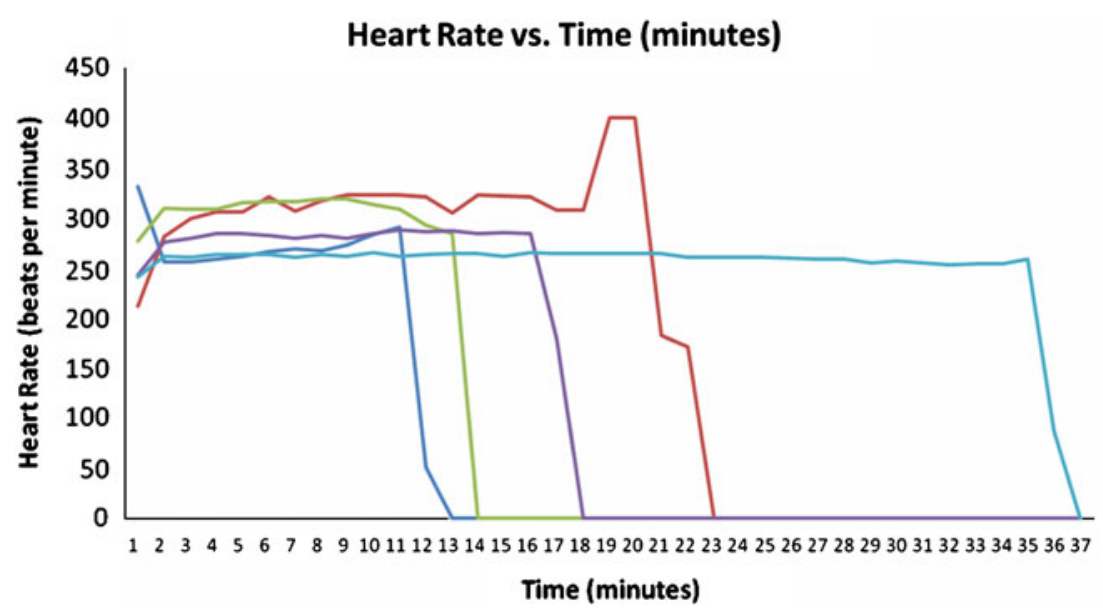


independent of and additive to beta-adrenergic stimulation [12].

Jeevandam reviewed three separate studies of $\mathrm{T} 3$ on donor and recipient cardiac function in the setting of heart transplant and found that $\mathrm{T} 3$ decreased both the amount and duration of inotropic support [16]. A randomized, prospective trial by Sirlak et al. studied 80 patients undergoing CABG surgery who were pretreated with T3 for 7 days prior. Patients in the treatment arm were found to have decreased chronotrope, inotrope, pressor, and intra-aortic balloon pump requirements following surgery as well as having higher cardiac indexes [17]. Another prospective CABG study showed that patients treated with $\mathrm{T} 3$ after cross-clamp removal demonstrated improved postoperative ventricular function, reduced need for inotropic agents and mechanical devices, and decreased incidence of myocardial ischemia [18].

One study performed since the completion of our investigation suggests a potential deleterious effect of thyroid hormone in verapamil toxicity. Sokhanenkova et al. demonstrated effective treatment of calcium-induced arrhythmias with lower doses of verapamil in both hypoand hyperthyroid rats compared to controls [22]. These data imply increased myocardial sensitivity to both calcium salts and verapamil in the setting of thyroid hormone dysfunction in which case triiodothyronine therapy could potentiate verapamil toxicity. Our study found no increase in toxicity to support increased myocardial sensitivity to verapamil during T3 treatment.

Despite previously demonstrated benefits of T3 therapy for hypotension of nontoxic etiology, continuous infusion of T3 did not result in significant differences in survival, heart rate, or MAP in this pilot study of severe verapamil poisoning. Several explanations for the lack of effect may be considered. The most obvious is that $\mathrm{T} 3$ activity offers no hemodynamic benefit at any time following $\mathrm{CCB}$ intoxication. More likely, the time frame of this study limited the demonstration of a potential effect. Thyroid hormone acting via nuclear receptors induces synthesis of calcium adenosine triphosphatase of the sarcoplasmic reticulum, alpha-myosin heavy chain, and the $\mathrm{Na}^{+}, \mathrm{K}^{+}$ ATPase [8-10]. These effects would not be immediate due to the time necessary for gene expression and protein synthesis. Mortality in this study was rapid $-23 \mathrm{~min}$ in the control and $30 \mathrm{~min}$ in the study group. In clinical scenarios, progression of toxicity occurs over several hours due to gastrointestinal absorption which may afford critical time for T3 to work. The protocol for this study, however, was designed to detect immediate vasopressor effects to mimic emergent treatment of the poisoned patient.

In addition, the goal of this study was to evaluate T3 monotherapy. The role of $\mathrm{T} 3$ in myocyte senstization to beta-adrenergic stimulus may provide synergistic activity with vasopressor co-administration. However, potential synergistic benefits were not investigated in this pilot study.

Another limitation was the degree of cardiovascular toxicity induced. Dose-finding experiments showed that discontinuation of verapamil infusion once desired toxicity was achieved resulted in gradual spontaneous improvement in the hemodynamic status of the animals. Our goal was to evaluate the use of T3 as monotherapy in severely poisoned subjects who would theoretically perish without intervention. However, our protocol led to very rapid deterioration in blood pressure that may have masked subtle beneficial effects of $\mathrm{T} 3$.

This investigation was further limited by the small number of study subjects permitted by the Institutional Animal Care and Use Committee. No more than ten animals may be used to conduct pilot research. Small changes between groups may not have been detected.

Further research would need to be conducted to evaluate efficacy of T3 therapy over a longer duration of toxicity as well as in conjunction with other standard therapies. The physiologic function of $\mathrm{T} 3$ as well as experience in other patient populations would indicate potential benefit following CCB toxicity, but none was found in this study.

\section{Conclusion}

Despite rapid escalation of $\mathrm{T} 3$ infusion, no effect on survival, heart rate, or MAP was found in rats following severe verapamil intoxication. Triiodothyronine monotherapy offers no significant short-term improvement over normal saline in a rat model of verapamil toxicity.

Acknowledgments Funding for this experiment was provided by the following research grants: the Pittsburgh Emergency Medicine Foundation and American College of Medical Toxicology Jazz Pharmaceuticals Research Award. The results of this study were presented at the 2008 North Amercian Congress of Clinical Toxicology as a poster presentation and abstract.

\section{References}

1. DeWitt CR, Waksman JC (2004) Pharmacology, pathophysiology, and management of calcium channel blocker and beta blocker toxicity. Toxicol Rev 23(4):223-238

2. Ramoska EA, Spiller HA, Winter M et al (1993) A one-year evaluation of calcium channel blocker overdoses. Toxicity and treatment. Ann Emerg Med 22:196-200

3. Bailey B (2003) Glucagon in beta-blocker and calcium channel blocker overdoses: a systematic review. J Toxicol Clin Toxicol 41 (5):595-602

4. Kline JA, Raymond RM, Schroeder JD et al (1993) Insulin is a superior antidote for cardiovascular toxicity induced by verapamil in the anesthetized canine. J Pharmacol Exp Ther 267:744-750 
5. Yuan TH, Kerns WP II, Tomaszewski CA et al (1999) Insulinglucose as adjunctive therapy for severe calcium channel antagonist poisoning. J Toxicol Clin Toxicol 37:463-474

6. Bania TC, Chu J, Perez E, Su M, Hahn IH (2007) Hemodynamic effects of intravenous fat emulsion in an animal model of severe verapamil toxicity resuscitated with atropine, calcium, and saline. Acad Emerg Med 14(2):105-111

7. Tebbutt S, Harvey M, Nicholson T, Cave G (2006) Intralipid prolongs survival in a rat model of verapamil toxicity. Acad Emerg Med 13(2):134-139

8. Danzi S, Klein I (2004) Thyroid hormone and the cardiovascular system. Miverva Endocrinol 29(3):139-150

9. Dillman W (2002) Cellular action of thyroid hormone on the heart. Thyroid 12(6):447-452

10. Shao Y, Ojamaa K, Klein I et al (2000) Thyroid hormone stimulates $\mathrm{Na}, \mathrm{K}$-ATPase gene expression in the hemodynamically unloaded heterotopically transplanted rat heart. Thyroid 10(9):753-759

11. Timek T, Vahl CF, Bonz A et al (1998) Triiodothyronine reverses depressed contractile performance after excessive catecholamine stimulation. Ann Thorac Surg 66(5):1618-1625

12. Walker JD, Crawford FA Jr, Mukherjee R et al (1994) Direct effects of acute administration of 3, 5, 3' triiodo-L-thyronine on myocyte function. Ann Thorac Surg 58(3):851-856

13. Zuppa AF, Nadkarni V, Davis L et al (2004) The effect of a thyroid hormone infusion on vasopressor support in critically ill children with cessation of neurologic function. Crit Care Med 32 (11):2318-2322
14. Salim A, Vassiliu P, Velhamos GC et al (2001) The role of thyroid hormone administration in potential organ donors. Arch Surg 136 (12): $1377-1380$

15. Jeevandam V, Todd B, Regillo $T$ et al (1994) Reversal of donor myocardial dysfunction by triiodothyronine replacement therapy. J Heart Lung Transplant 13(4):681-687

16. Jeevandam V (1997) Triiodothyronine: spectrum of use in heart transplantation. Thyroid 7(1):139-145

17. Sirlak M, Yazicioglu L, Inan M et al (2004) Oral thyroid hormone pretreatment in left ventricular dysfunction. Eur J Cardiothorac Surg 26(4):720-725

18. Mullis-Jansson SL, Argenziano M, Corwin S et al (1999) A randomized double-blind study of the effect of triiodothyronine on cardiac function and morbidity after coronary bypass surgery. $\mathrm{J}$ Thorac Cardiovasc Surg 117(6):1128-1134

19. Tuncok Y, Apaydin S, Gelal A, Ates M, Guven H (1998) The effects of 4-aminopyridine and Bay K 8644 on verapamil-induced cardiovascular toxicity in anesthetized rats. J Toxicol Clin Toxicol 36(4):301-307

20. Machin D, Campbell M, Fayers P, Pinol A (1997) Sample size tables for clinical studies, 2nd edn. Blackwell Science, Malden

21. Zar JH (1984) Biostatistical analysis, 2nd edn. Prentice-Hall, Englewood Cliffs

22. Sokhanenkova AE, Sokhanenkov MI, Afanas'eva EI, Arzamastsev EV (2008) Characteristics of pharmacological and toxic effects of verapamil during cardiac arrhythmia in thyrotoxic and hypothyroid rats. Kardiologiia 48(6):57-61 